\title{
Integrated monitoring can be effective in tiger conservation
}

\section{Opinion}

Decline in number of tigers around the world has prompted range countries for setting up goal to double tiger population by year 2022 . However lack of scientific data about the tiger population is a major problem. Now Indian researchers have found in a study that integrated monitoring could be helpful in tiger conservation. Researchers wanted to know that how the tiger population is affected by variation in habitat quality. Study was conducted with camera trap method in Beligiri Rangaswamy Temple tiger reserve in Karnataka province of India. Camera traps were deployed at 157 locations during March and May 2015. A total of 535 tiger photographic 'captures' were obtained from the camera trapping efforts. Researchers have estimated 55 tigers in Rangaswamy Tiger Reserve. The average number of tigers in the area found to be more than six in per 100 square kilometers area of the tiger reserve.

In camera trap survey method hundreds of camera traps placed on the paths used by tigers in forest. These censor-based camera capture pictures of tigers when they come in the range of them. However, this method persists some problems also, such as possibility of repeated photo capture of a tiger on different locations. Captured Images were sorted on the basis of wildlife species and individual tigers were identified using the Wild-ID, a pattern recognition software, to match tiger stripes and cross-checked manually, after which every individual tiger was given a temporary identification number as prescribed under the National Tiger Conservation Authority (NTCA) protocol. "Wildlife population often found to be higher in high quality natural habitat. But the abundance of a particular species at one site too can be a threat for their existence. In such a situation, animals start migrating to adjacent low-quality habitat. Although animals has to struggle there too if the habitat quality is not enough good for their existence", Say Sanjay Gubbi, ${ }^{1}$ member of the study group. Gubbi is renowned Indian environmentalist associated with Nature Conservation Foundation based in Mysore, India.

Rangawamy tiger reserve is part of a larger landscape consisting of other protected areas including Malai Mahadeshwara Hills and Cauvery Wildlife Sanctuary, Bannerghatta National Park in Karnataka, and Satyamangalam Tiger Reserve and North Cauvery Wildlife Sanctuary in Tamil Nadu. Ragaswamy reserve hosts a diversity of habitats within its boundaries including evergreen and semi-evergreen forests, dry and moist deciduous forests, and scrub growth. According to Gubbi, "It's important to scientifically monitor tiger numbers in all tiger source sites which we have tried to do at Ragaswamy reserve. It also provides an opportunity to understand the source-sink dynamics

\author{
Volume 2 Issue 5 - 2017 \\ Umashankar Mishra,' Priyanka Mishra² \\ 'India Science Wire, India \\ ${ }^{2}$ ICAR-National Bureau of Animal Genetic Resources, India
}

Correspondence: Priyanka Mishra, ICAR-National Bureau of Animal Genetic Resources, Haryana, India, Emailmiraclepriya@gmail.com

Received: November 26, 2017 | Published: December 0I, 2017

as Rangaswamy Tiger Reserve itself is nestled within a larger tiger landscape."

"This study suggests exchanging policy and management information across the landscape as there are four protected areas and other reserved forests within the landscape. A very important point would be to gazette North Baragur, South Baragur, Biligundlu, Taggatti, Bandavadi and other reserved forests that are in Tamil Nadu and adjoins Rangaswamy Tiger Reserve and MM Hills Wildlife Sanctuary as part of a wildlife sanctuary", say Gubbi. Apart from Sanjay Gubbi, study team includes Rashmi Bhatt of Nature Conservation Foundation and Ling raj \& Swayam Chaudhary ${ }^{2}$ of Forest Department of Karnataka. Study was recently published in research journal Current Science. ${ }^{3}$

\section{Acknowledgements}

None.

\section{Conflict of interest}

The author declares no conflict of interest.

\section{References}

1. Gubbi S, Nagashettihalli H, Kolekar A, et al. From intent to action: a case study for the expansion of tiger conservation from southern India. Glob Ecol Conserv. 2017;2017(9):11-20.

2. Lingaraja SS, Swayam Chowdhary, Rashmi Bhat, et al. Evaluating a survey landscape for tiger abundance in the confluence of the Western and Eastern Ghats. Current Science. 2017;113(9):1759-1763.

3. Bolger DT, Morrison TA, Vance B, et al. A computer-assisted system for photographic mark-recapture analysis. Methods in Ecology and Evolution. 2012;3:813-822. 